\title{
Application of Signal Detection Theory to Ascertain Driving Risk and Mitigation in a Flooded Urban Area
}

\author{
Eunsu Lee ${ }^{1}$, Kambiz Farahmand ${ }^{2}$ and Peter G. Oduor ${ }^{3}$ \\ 1. Upper Great Plains Transportation Institute, North Dakota State University, Fargo, ND58105, USA \\ 2. Department of Industrial and Manufacturing Engineering, North Dakota State University, Fargo, ND58105, USA \\ 3. Department of Geosciences, North Dakota State University, Fargo, ND58105, USA
}

\begin{abstract}
Signal Detection Theory (SDT) offers an unparalleled deterministic set of decision variables necessary to formulate applied risks in transportation. SDT has distinct advantages over basic prediction models since the latter may not represent an entirely accurate analysis. Thresholds based on elements of stimulus (signal and noise) and response for: a Type I discrimination of response variable where decision outcomes and rates are computed for metacognition to discriminate a Type II of decision outcomes was set. We also adapted the classical Dijkstra's shortest path algorithm within a GIS environment using Avenue ${ }^{\circledR}$ programming. Contours derived from LiDARwere used to set flood levels while satellite imagery corresponding to Red River of the North inundated (signal) areas were acquired amongst other spatial datasets. The signal information was further dichotomized using a binary yes-no model. Origin and destination points constrained within Fargo-Morehead were generated using a random point generator. From these points, trips were generated with some connected segments traversing through flooded areas. By analyzing False Alarm Rate (FAR) and Corrected Rejection $(C R R)$ computation, we found out that, when Hit Rate $(H R)$ and FAR are both low then there was an increased corresponding sensitivity. At 30-35 ft flood level, the values for FAR and HR was 0.97 and 0.91 respectively. When $F A R>H R$, lower set flood levels offered numerous route choices. Corresponding routes with associated impedance can be classified for risk-averse drivers or risk-takers. While the risk-averse avoid risky and unfavorable routes, the risk-taker optimizes at an adjustment factor of $\omega=0.1$ or $\omega=0.2$. An idealistic stage is achieved for a conservative, $\omega$, equal to 0.4 or 0.5 , which indicates maximum achievement in terms of time gain and safety simultaneously. At $\omega=0.0$ the prevailing conditions can be considered unrealistic since they incorporate areas considered impassable with absolute resistance like segments with a "Road Closed" or "Detour" sign. The applicability of our approach can be used to design multi-level and multi-modal transportation systems involving risk.
\end{abstract}

Keywords: Impedance, false alarm rate, corrected rejection, signal, noise.

\section{Introduction}

Historically, the Red River of the North (predominantly located between the US states of North Dakota and Minnesota) is prone to major flooding with the year 2009 as the most recent record level. The last major flooding, back in 1997, was also as a result of heavy winter snowfall and early and sudden warming spring seasons resulting in excessive land saturation and extensive flooding [1]. The floodplain and surrounding areas has been repeatedly classified as a national emergency region by Federal Emergency

Corresponding author: EunSu Lee, PhD, GISP, CPIM, CSCP, research fields: transportation, supply chain management, logistics. E-mail: eunsu.lee@ndsu.edu.
Management Administration (FEMA) during the flood season.Flooding over roads and bridges were more prominent since water elevations of the flooded lands were higher than in the main drain channels [1]. Significant damage and travel retardants resulted along major portions of the valley. Simonovic and Ahmad [2] developed a dynamic system to capture human behavior during Red River flood emergency.The research was based on a post-flood survey and evacuation simulation using feedback processes theoretical framework.From their study, they found out that lack of knowledge is one of the reasons of loss of valuable time during an evacuation. They surmised that a high level of issuing warnings to the public and 
crucial timing of evacuation provided a better success. These two factors are based on policy variables of planning and operations.

Transportation systems play an important role in times of emergencies by providing evacuation gateways to stranded communities. In addition, road and bridge closures in a flooded area disrupt travel and freight movement. The Department of Transportation (DOT) and other transportation authorities are delegated with the mandate to determine detours and set alternate routes circumventing flooded areas. This information is critical to emergency responders and risk-mitigation planners. In many cases due to the dynamic characteristics of a flood, accurate and up-to-date information may not be readily available. The public in most cases would collect information from private and other sources, such as media or rely on personal experiences.

When users choose a route, they use their personal experiences and intuition about travel time and conditions of the routes. Unexpected road conditions and closures make driving more difficult and the traffic generate a lot of frustration due to the unexpected road closures and constructions.Drivers may not know exactly which path is safe because of a lack of knowledge and information about road conditions and water depth [3]. Drivers also may travel more secured routes to avoid risky places or heavy traffic conditions. In fact, most drivers will usually do a quick risk analysis and determine their ad-hoc risk tolerance. To deal with the routing choices under uncertainty, a variety of techniques are used by adaptive neuro-fuzzy inference mixing artificial neural network and fuzzy logic [4, 5], Bayesian logics [6], and simulation. Signal Detection Theory (SDT) has been applied to (a) ascertain hazard perceptions in collisions, for example, Parasuraman et al. [7] (b) analyze differences between trained and novice drivers, for example, Wallis and Horswill [8], (c) to set aviation safety zones [9].

Geographic Information Systems (GIS) with Digital Elevation Models (DEM) derived from remotely sensed stereoscopic data can be utilized to predict the high likelihood areas that would be flood prone and affected routes. Real-time geospatial information may also provide users and planners visual information and an unbiased rendition of dynamic and fast changing situations. The main objective of this study was to design a route choice approach with impedance, which takes into account both travel time and user perceptions.

\subsection{Study Area}

The main area of focus for this study is Fargo (North Dakota)-Moorhead (Minnesota) metropolis along both banks of the Red River. We used historic flood data and 2009 flood events that occurred in March and April for this research. Significant flooding in 2009 occurred also further downstream resulting in closure of the interstate highway I-29 between North Fargo and Hillsboro. Bridges over the interstate highway I-94 were open however, connecting regions were not recommended for public access. Another connecting interstate highway, I-94 was closed on March 28, 2009 when the flood level was at 40.65 feet. The Red River floodplain is almost plain and low which further compounded the problem from flooded rural roads to overflowing sewage and storm drain systems. Due to unpredictable weather conditions and breached temporary levees, travel information was unreliable, insofar as flood stage and road closure information was available through media and pertinent websites.

\section{Theory}

\subsection{Basic Parameters}

Unexpected events like flooding directly impact the economy and hurt public sector's transportation [10]. Shippers and carriers need accurate, timely information and communication system for pre- and post-disruptions. To analyze supply chain vulnerabilities, the following parameters are highly necessary: (1) mechanism of changes caused by disruptions becomes a vital parameter. (2) Another 
parameter necessary in geospatial analyses includes level of water depths on streets [3]. (3) Normalized negative and positive feedbacks based on residents' perceptions and experiences based on (i) phases of concern, (ii) danger recognition, and (iii) evacuation decision [2]. (4) An accessibility index for link disruptions caused by flood damages [11]. Secondary parameters include (i) distance decay function and (ii) traffic volume for multi criteria decision for the accessibility measurement, and (iii) dynamic effects of the period for recovery. Here the probability of flood damage on a link in 100-year period is used to adjust the expected value of accessibility deterioration for a disrupted links.

2.1.1 Resistance, Reactance and Congestion

The value of impedance (called disutility), $X$, is the sum of two components, $X=X_{g}+X_{R}$. For the total impedance, two types of in-vehicle times can be mentioned: (1) general (basic) impedance, $X_{g}$, which is based on inevitable, value-added transportation activities, and (2) the tardiness (redundant) impedance, $X_{R}$, based on unvalued-added activities $[12,13] . X_{g}$ is calculated in the basic impedance, of which tardiness impedance is equivalent to null under an ideal status. This type of impedance in transportation is a necessary commute connecting products or services to consumers or users. Impedance can also be calibrated in terms of monetary or time values.

Oak Ridge National Laboratory [14] developed an impedance equation to estimate the mode-specific travel distance based on origin-destination (OD) matrix from commodity flow survey (CFS) 2000 conducted by Bureau of Transportation Statistics (BTS) and U.S. Census Bureau for industry survey. The impedance [15] is expressed as,

$$
R_{s}=\left(L_{s} \times r_{s}\right)+f_{s},
$$

where $R_{s}$ is impedance value, $L_{s}$ is length of a segment, called basic link impedance, $r_{s}$ is relative link penalty based on type of mode and road conditions, and $f_{s}$ is link penalty not related to $L_{s}$. From Eq. (1), two different types of impedance values are used (1): one is ratio penalty to the length $\left(L s \times r_{s}\right)$ called resistance [16] and the other is penalty score $\left(f_{s}\right)$ also called reactance. The speed limit based on highway capacity manual [17] can be used for input speed information. The penalty impedance can be determined using mode equilibrium.

Deterministic impedance models adopt a Stochastic User Equilibrium model (SUE) for Freight Analysis Framework (FAF) [18]. These can be adopted for route choice and impact analysis in studying response tonatural disasters. Modifying Eq. (1) and incorporating the total impedance for a path yields:

$$
T_{s}=\left(\frac{L_{s}}{V_{s}} \times r_{s}\right)+f_{s}
$$

Sum of all link impedances can be denoted by, $\sum_{s \in P} T_{s}$ where $s$ is a link of a selected path $P$.An adjusted $r_{s}$ can also be estimated from $\prod_{i \in N} r_{s i}$ where $r_{s i}$ represents elemental resistance of a segment.Free flow speed, $V_{s}$, for a segment can be obtained from NCHRP Report 387.

Due to preference of safe and quick delivery, congestion becomes an important factor since this will directly impact delivery times.In a decision support system, when the best routes are termed busy due to preferential assignment, other alternatives are programmatically reviewed and assigned for subsequent trips. The Bureau of Public Roads (BPR) congestion functioncan be used by adopting the adjusted travel time impedance. In this study, congestion resistance model was derived as:

$$
V C_{s}=\alpha\left(\frac{V_{s}}{C_{s}}\right)^{\beta}
$$

where $V C_{\mathrm{s}}$ is volume-to-capacity ratio, $V$ is traffic volume on a link, $C$ is practical capacity of a link, and $\alpha$ and $\beta$ are adjustment factors. Congestion can therefore be recalculated for each trip assignment on network links. In this study, $\alpha$ and $\beta$ were set at 0.15 and 4.0 respectively. When $\alpha=1.0$, half of the free flow speed would be achieved at a volume/capacity ratio of 1.0. 


\subsection{Impedance-Pertinent Multi-Criteria Approach}

The basic approach used here was derived by decomposing an adjusted travel time with respect to resistance and reactance.Resistance and reactance as stipulated in this study can be adjusted using an adjustment factor, $\omega$, within an adjusted travel time, $T_{P}^{\text {adjusted }}$, and a normal time, $T_{P}^{\text {normal }}$. Risk-averse travelers $(\omega=1)$ would require $T_{P}^{\text {adjusted }}$ since we deem that they would respond conservatively to the most flooded regions from available information. On the other hand, risk-takers would resort to the shortest travel time route based on $T_{P}^{\text {normal }}$ if and only if $\omega \rightarrow 0$ whereas $\omega=1.5$ for risk-neutral travelers. Total impedance, $T_{z p}(\mathrm{Ohm})$, can be minimized from:

$$
T_{Z P}=\omega \cdot\left(T_{P}^{\text {adjusted }}\right)+(1-\omega) \cdot T_{P}^{\text {normal }}
$$

where $T_{z p}$ is impedance of a predicted path $P$, which is predicted.We can redefine the general concepts of impedance, $Z$, in route choice by further subdividing it into resistance, $R$, and reactance, $X$. Resistance, $R$, can therefore be viewed as a factor that prevents ideal mobility free of underlying constraints and can be assigned a value between 0 and $1\left(R_{s}=[0,1]\right)$. Instead of a direct value of $R$, the complement of $R$ iswas used since the total resistance will be distorted when $\mathrm{R}=0$. Resistance on a link can be represented as a multiplication form of the complement designated as $R_{s k}$ and affected by a $k$ factor possibility. The following set of equations can be used to gauge penalty on a weakly connected segment [19]:

$$
\left\{\begin{array}{l}
R_{s}=1-\left(\prod_{k=1}^{K} \bar{R}_{s k}\right) \\
v_{s}^{\text {adjusted }}=v_{s} \times R_{s} \\
L_{s}^{\text {adjusted }}=L_{s} \times \mathfrak{R}_{s} \\
T_{P}^{\text {adjusted }}=\frac{L_{s \in P}^{\text {adjusted }}}{v_{s \in P}^{\text {adjusted }}}
\end{array}\right.
$$

The total utility value $\left(\prod_{k=1}^{K} \bar{R}_{s k}\right)$ is subtracted from one. To assign a penalty on a weakly connected segment penalty, the travel time, $T_{P}^{\text {adjusted }}$, is adjusted by the resistance under the assumption that users' perceptions for traversing the link affects the link travel speed, $v_{s}^{\text {adjusted }}$, equivalent to traveling a longer link length, $L_{s}^{\text {adjusted }}$. The adjusted speed on segments, which is a set of the optimal path, is used to compute the total travel time, $T_{P}^{\text {adjusted }}$. The major issue of impedance is determining and calibrating $k$ from Eq. (5). In our case however, some of the elements are highly correlated while most are independent as such we can assume an overall interdependency. Let $E$ be a set of elements such that, $E=\{$ Road classification, River buffer, Elevation, Congestion $\}$. The first element, road classification, based on the HCM [17] can be used to estimate a free-flow speed value. The next two elements (River buffer, Elevation) can be calibrated and predicted using a LiDAR data set with elevation information and satellite imagery utilized to quantify extent of inundation. The last element, (Congestion) can be measured using a BPR function and periodically updated after trip assignment of any selected route.

\subsection{Signal Detection Theory (SDT)}

Signal Detection Theory (SDT) has been applied to various areas with agreeable success rates [20, 21]. SDT, which represents the deterministic static evaluation, in our study, was applied to River buffer and Elevation elements. SDT is adopted to represent users' perceptions for flood zones especially in the presence of uncertainty. This can be generated by a known process (signal) and change process (noise) [21]. Fig. 1 illustrates a simplistic version of SDT. From Fig. 1, the dashed polygon represents inundated area as assessed. The dark polygon represents a buffer zone, created along the river based on a user's perception. Prediction models may not represent accurate information at all times to cover an entire region. In Fig. 1, the response region $(S)$ is delineated by a 0.1 mile buffer in response and represents about 350 $(30 \mathrm{~m} \times 30 \mathrm{~m})$ grid cells, while the flooded region $(s)$ is only 250 grid cells. 500 out of 750 cells were not inundated. User response to no flood $(N)$ regions is 400 out of 750 cells (Table 1). Based on a user's perception represented by the response buffer polygon, the 
Table 1 Derived probability values.

\begin{tabular}{|c|c|c|c|}
\hline \multirow{2}{*}{ Reality } & \multicolumn{3}{|c|}{ Response } \\
\hline & Yes $S$ (Flood) & No $N$ (No Flood) & Total \\
\hline $\begin{array}{l}\text { Signal Present s } \\
\text { (Flood) }\end{array}$ & $\begin{array}{l}\text { Hit } \\
\text { No. of }\{\mathrm{Hit}\}=200 \\
\mathrm{HR}=0.8\end{array}$ & $\begin{array}{l}\text { Miss } \\
\text { No. of }\{\text { Miss }\}=50 \\
\text { MR }=0.2\end{array}$ & $\begin{array}{l}s=250 \\
1.0\end{array}$ \\
\hline \multirow[t]{2}{*}{$\begin{array}{l}\text { Signal Absent } \mathrm{n} \\
\text { (No Flood) }\end{array}$} & $\begin{array}{l}\text { False Alarm (FA) } \\
\text { No. of }\{\text { FA }\}=150 \\
\text { FAR }=0.3\end{array}$ & $\begin{array}{l}\text { Correct Rejection (CR) } \\
\text { No. of }\{\mathrm{CR}\}=350 \\
\mathrm{CRR}=0.7\end{array}$ & $\begin{array}{l}n=500 \\
1.0\end{array}$ \\
\hline & $\begin{array}{l}R_{\mathrm{Y}}=350 \\
r_{\mathrm{S}}=1.0\end{array}$ & $\begin{array}{l}R_{\mathrm{N}}=400 \\
r_{\mathrm{N}}=1.0\end{array}$ & 750 \\
\hline
\end{tabular}

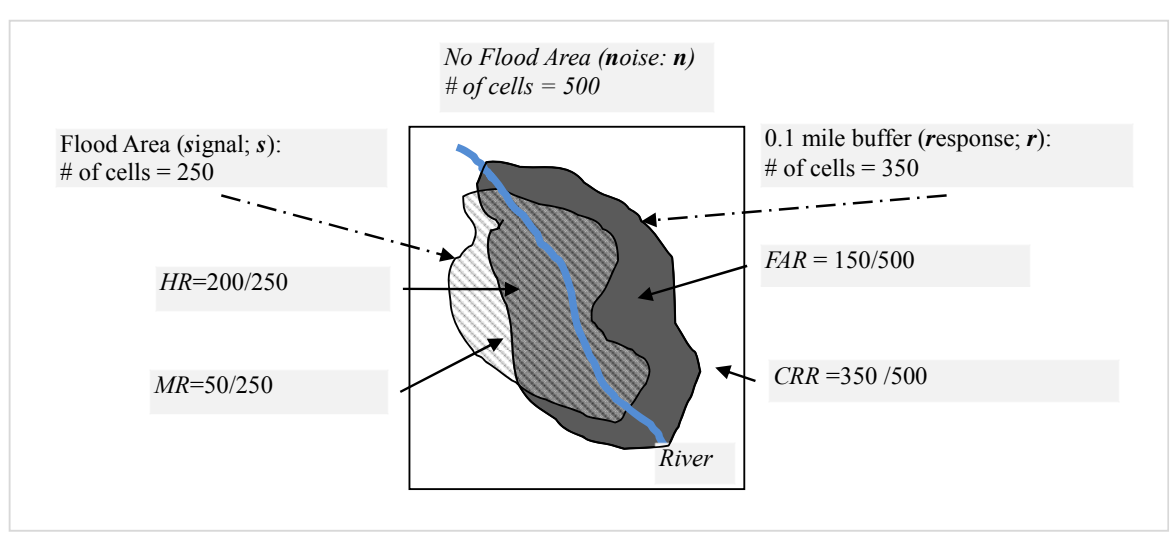

Fig. 1 Schematic representation of Signal Detection Theory in a flood region.

probability of a "Yes" decision is $0.8(=200 / 250)$. Onthe contrary, the conditional probability of Yes (S) decision given non-flood region (n) is $0.3(=150 / 500)$. In an SDTscenario, buffer zones are therefore used to estimate "flood warning regions" using historical inundation data such as 500- or 100-year floodplains. In our study, we set thresholds based on elements of stimulus (signal and noise) and response for: a Type I discrimination of response variable as defined in Step 1 where decision outcomes and rates are computed for metacognition to discriminate a Type II of decision outcomes as defined in Step 2 below. Sensitivity and validation can be conducted thereafter [9, 22].

2.3.1 Step 1: Signal and Noise; Response

All possible states of the world (SoW) were assigned using non-overlapping curves [23]. The environmental hazard, for example, was detected using a dichotomous categorical state of flood (signal) and no flood (noise).The signal in our study represented hazard areas in cell units, which can be changed over time based on water level fluctuations caused by a variety of exogenous factors (e.g., rain, snow melting speed, and saturation) and endogenous factors (e.g., flood protection efforts of building a dike, sandbagging, and building a diversion channel) [7]. When drivers are not convinced about the flood hazard, they may set their own safety regions in the no flood regions as a noise (non-signal) data set. In addition, we identified internal noises as variations of Region of Interests (ROI), software platform conversion, datum conversion; external noises were time variation over time, water fluctuation by environmental factors, and flood protection. We are also aware that it is virtually impossible to map inundation perfectly since external noises are not controllable.

Response can be categorized by a Yes $(Y)$ or a No (N) judgment. In this study, Yes $(Y)$ implied that travelers would consider the inundation benchmark information and select to travel through flooded or non-flooded areas (see Fig. 1). From Fig. 1, the mapping function of response, $r$, is based on perceived severity, criticality, and intensity [15]. We also constrained the signal and 
noise zones based on a real-time response region necessary for the driver to make critical decisions based on degree of flooding, road closures, and/or severe danger (Fig. 1). Response was assigned based on an "evidence variable" for example inundation imagery. The response was coded as 0 or 1 , for example, for a flooded area the response was set to 1 and 0 otherwise.

\subsubsection{Step 2: Decision Outcomes Using of} Implication Functions

There are two types of metacognitive judgment responses to Flood and No Flood reality. Each event falls in one of the four elemental categories from the combination of signal-noise in the reality column and Yes-No in the response row (see Table 1). Our approach was to search for a reliable route if a driver chose to travel through the region especially if they had prior knowledge of areas with flood warning posting. A "Yes" response to the signal, $s$, indicates an acknowledgement of flooding hereby referred to as a Hit $(\mathrm{H})$. On the other hand, a "Yes" response to No Flood situation is designated as a False Alarm (FA). A "No" response to Signal Absent, $n$, reality of "No Flood" is also a correct decision, termed Correct Rejection $(C R)$; however, a "Yes" response to "No Flood" reality becomes a wrong decision, also referred to as a Miss (M).

2.3.3 False Alarm Rate $(F A R)$ and Corrected Rejection (CRR) Computation

The following sets of probabilities are deemed as necessary integral components of SDT:

$$
\begin{gathered}
H R=P(S \mid s) \\
M R=P(N \mid s) \\
C R R=P(N \mid n) \\
F A R=P(S \mid n)
\end{gathered}
$$

where probability of a Hitfrom Eq. (6a), $H R$, is the ratio of the intersection between the flooded area and each buffer river to the flooded region, missing ratefrom Eq. (6b), $M R$, is the ratio of the intersection of the No Flood area and each river buffer to the Flood region. The probability of Correct Rejectionfrom Eq. (6c), $C R R$, represents a Yes response when the signal as identified in Step 1 is not present while the probability of False Alarmfrom Eq. (6d), FAR, is the ratio of the non-flooded area subtracted by the overlapping between the flooded area and the buffer to the non-flooded area. Only two of four probabilities are needed since $H R+M R=1$ and $C R R+F A R=1 . H R$ and FAR probabilities can then be used to compute performance measurements in response to signals [20]. We assume that the noise variables are normally distributed and the two distributions have an equal variance. Our goal in this kind of computation is to assess the risk based on driver tendencies, as such the gain is not the main issue as much as associated impedance due to flooding. The resistance to impedance constitutes relative flow retardants of the signal to noise stemming from the response region. We can envision a scenario where a driver attempts to travel through an area designated as flood hazard area with an implied confidence of no flooding or he/she ignored $H R$ value. On accessing an impassable region, there is no other option other than turning back to the starting point or taking the nearest detour costing $\mathrm{him} /$ her lost time and nonetheless incurring other travel risks. In contrast, if a driver decides to take a longer detour route by assuming a higher confidence due to likelihood of possible flooding when the region actually is not floodedthen this becomes the classical $F A R$ and will cost the driver lost time.

The conversion of non-parametric values $H R$ and $F A R$ to time dimension requires normalization. To effect this, the likelihood of incurred resistance in navigating through the response region can be expressed by $l_{b}=C(H) / C(F A)$, where $C(H)$ is cost of making a hit, taking the associated risk and $C(F A)$ denotes cost of triggering a false alarm [20, 21]. If the cost of the hit and false alarm are the same, $l_{b}$ can be substituted by

$\{[C(H) \times P(S \mid s)] /[C(F A) \times P(S \mid n)]\}=P(S \mid s) / P(S \mid n)=H R / F A R$

In Table 1 , for $l_{b}=0.8 / 0.3=2.67$ which implies that the roads located within the buffer zone will have a 
distance resistance factor $(\mathfrak{R})$ of 2.67 longer than for the roads in "No Flood" area. Time loss can be secondarily determined from Eqs. (2) and (5).

\section{Methodology}

We adapted the classical shortest path algorithm within the GIS environment since this helps minimize total disutility. ArcView ${ }^{\circledR} 3.3$ with Avenue $^{\circledR}$ programming language was used to develop custom menu, tools, and buttons for parameter entry, validation rules, and data processing. Throughput on links served as the amount of network flow that is served over a period [24]. In this study, capacity-constrained trip assignment was doneusing dynamic programming where input can be derived from ASCII data or from users through a graphic user interface (GUI) form. The modular nature of Avenue ${ }^{\circledR}$ programming allows formulated functions to displayas tables or forms all associated cost factors such as distance, travel time, and impedance automatically. The overall goal of this level of programming is to determine assigned trip and total traffic information for each network segment. Each trip to- and from- nodes was randomly generated using a random point generator using Spatial Analyst extension in ArcMap-Arc/INFO ${ }^{\circledR} 9.3$.

A Digital Elevation Model (DEM) for the study site derived from Light Detection and Ranging (LiDAR) datasets was obtained from International Water Institute (IWI). Smoothed 1-foot contours for the region were also acquired from IWI. Polygons representing elevation categories representing flood levels $0-30$ feet, 30-35 feet, 35-40 feet, and above 40 feet were generated using basic spatial analyses. The flood levels were adjusted to reflect altitudes referenced to sea level datum. Satellite imagery from LANDSAT -5 for the following dates: (1) March 26, 2009 (with cloud cover of 99\%), (2) April 11, 2009 (with cloud cover of 1\%), (3) March 18, 2009 (with cloud cover of 7\%), (4) and April 3, 2009 (with cloud cover of $38 \%$ ) were acquired. This data comes with only asystematic correction of Level $1 \mathrm{G}$ (radiometric and geometric only). Road construction maps and historic road closures data was obtained from North Dakota and Minnesota Departments of Transportation. Road networks were obtained from Tiger/Line ${ }^{\circledR}$ geospatial portalhosted by U.S. Census Bureau since it includes all detail road segments in the study region including connecting country roads and ramps. National bridge inventory data used to ascertain bridge closures was obtainedfrom Federal Highway Administration (FHWA).

The following U.S. Census Bureau's Master Address File/TIGER feature class codes (MTFCC) were derived from TIGER $^{\circledR} /$ Line data, (1) S1100 for interstate highways, (2) S1200 for U.S./state highways, (3) S1400 for country roads, and (4) S1740 for interstate highway ramps (see also Table 2). Speed limit information was derived based on HCM [17] guidelines. For each road class, associated impedance was assigned based on HCM speed adjustment weights (Table 2). For interstate highways in rural and urban areas, a basic resistance value was assigned even though the speed limit varies in status and by functional class. We also considered a parking lot as also a passable segment at a lower speed. Higher impedance values were assigned for (a) road medians in order to employ a prohibitive factor, and (b) ramps to generate slower speeds necessary for safe turn negotiations.

The three buffers from Red River were generated for the empirical study (Fig. 2). Confidence categories were set based on buffer distances $(0.1,0.3,0.5$ miles, and over 0.5 miles). The " 0.1 mile" buffer represented a high flooding certainty and "over 0.5 miles" a low certainty. The buffer of over 0.5 miles was considered as basic impedance in terms of river buffer element. The signal value was set to a binary value of flood and non-flood. On the contrary, the response variable was continuous distance from a river to the boundary of the study region. The continuous spatial distance was grouped into buffers for ease of mapping aiding in determining extent of $s$ from Fig. 1. 
Table 2 Input values for resistance, speed, and free flow speed information from NCHRP 387.

\begin{tabular}{|c|c|c|c|c|}
\hline \multirow{2}{*}{ MTFCC in Tiger Lines } & Combination & Functional Class & \multirow{2}{*}{ Resistance } & \multirow{2}{*}{ Speed Limit (mile per hour) } \\
\hline \multirow{2}{*}{ S1100 (Primary road) } & \multirow{2}{*}{ AND } & Rural & 0.00 & 70 \\
\cline { 3 - 5 } S1200 (Secondary Road) & \multirow{2}{*}{ AND } & Urban & 0.00 & 55 \\
\cline { 3 - 5 } & & Rural & 0.12 & 65 \\
\hline \multirow{2}{*}{ S1400 (Local) } & \multirow{2}{*}{ AND } & Urban & 0.20 & 55 \\
\hline S1630(Ramp) & \multirow{2}{*}{ S1780 (Parking lot) } & Rural & 0.31 & 25 \\
\hline Otherwise & \multirow{2}{*}{} & Urban & 0.40 & 20 \\
\hline
\end{tabular}

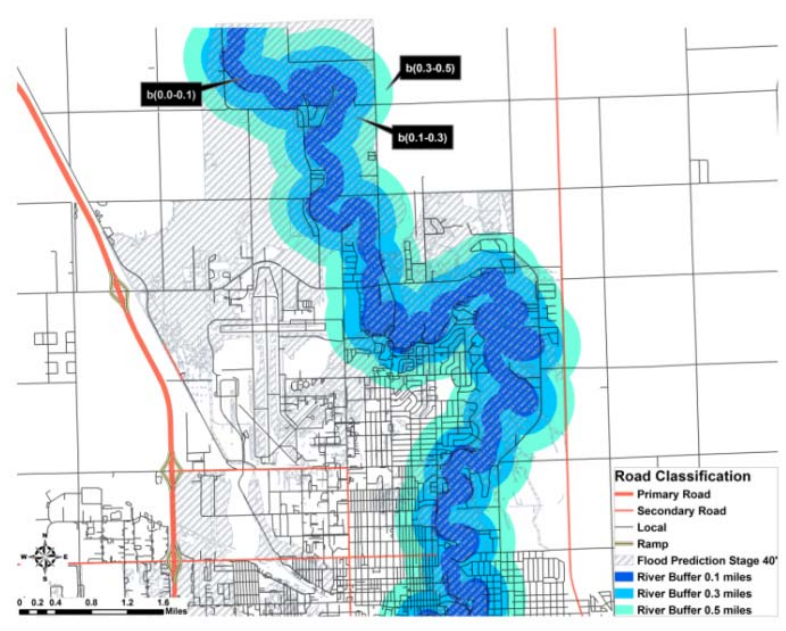

Fig. 2 Delineated buffer zones for the Red River study area.

The elevation was categorized into four groups:

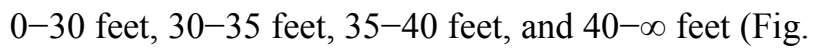
3). The 30 feet limit was selected as the commencement of flooding up to a crest level of 40 feet. Areas with elevation over 40 feet were classified as safe havens. The signal was set to a binary value of flood and non-flood, but the response variable was considered continuous from the river level of relative elevation 0 feet to the highest level in the regions. The continuous variable of the response required a mapping function to facilitate. The mapping function was determined using elevation, principally by initiating $h$, onto $s$. Levee and downstream-upstream separation, and protection efforts were not considered in the

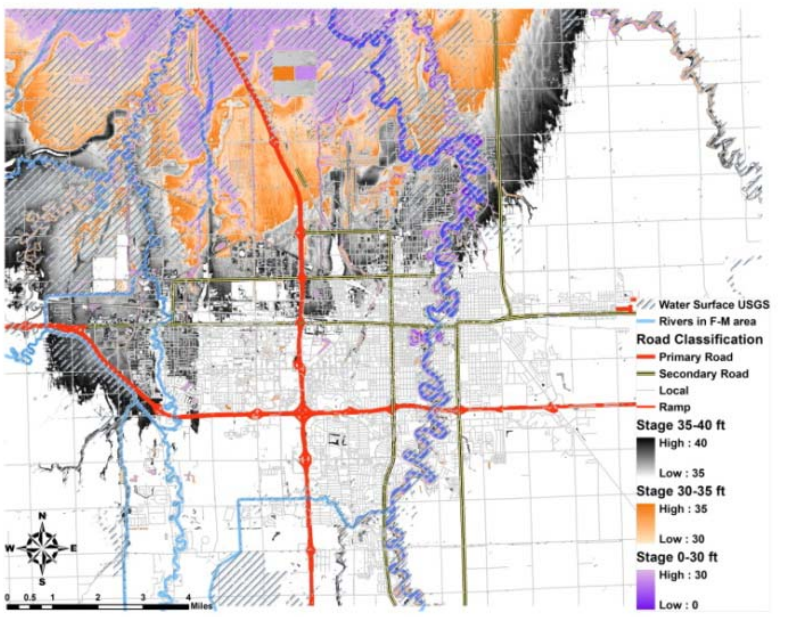

Fig. 3 Elevation levels along the study area.

elevation responses for the detailed inundation prediction.

The information relating bridge closures were transferred to road segmentswhere the bridges are located on as impedance. Bridges in areas designated as flooded were categorized as closed and flood caution information hard-coded on designated buffer zones. Speed limits were used to calculate the travel time through links using Eq. (5). From Eq. (5), the speed was adjusted based on the resistance, $R$, and reactance, $X$, values where the low possibility link throughput travel would be enforced by a lower travel speed. Theadjusted speed due to impedance factors was determined from:

$$
T_{s}^{\text {adjusted }}=\left(L_{s} \times \mathfrak{R}_{s}\right) /\left(v_{s} \times R_{s}\right)+X_{s}
$$


where $L$ is length, $v$ is speed, $R$ and $\mathfrak{R}$ are resistances, and $X$ a reactance. Here, $R$ is the resistance which relatively decreases the travel speed, while $\mathfrak{R}$ is the resistance which relatively increases the length of a segment. After determining this preliminary impedance for each segment we gauged performance mobility measured by the comprised combination of adjusted travel time and normal travel time by determining the lowest cost path using Spatial Analyst ${ }^{\circledR}$ extension between a designated origin and destination. This was done as a two-fold process, (1) using a time-space utility function [25] and (2) cost-distance function [26]. A penalty value (with time dimensions) was thereafter assignedbased on the lengthened distance weighted by $R$ and $X$.

The signal information was set using spatial polygons representing inundation. Confidence rating was thereafter mapped into dichotomous yes-no categories based on delineated polygons. The spatial random points were selected in the study areas using "Create Random Points" tool in Arc/Info ${ }^{\circledR}$ 9.3. Fifty five random points were generated in North Dakota (on the west side of Red River) as trip origins and forty five points for Minnesota (on the east side of the Red River) as trip destinations. For multi-criteria choice model, $\omega$ from Eq. (3) was constrained to a value between 0.0 and 1.0 by setting the random number generator within the set limits.

\section{Results and Discussion}

Possibility of flooding extending to the buffer regions was estimated based on the Signal Detection Theory (SDT) (Table 3). For rate scaling, we set $R\left(\theta_{b(n)}\right)=[0,1]$ and $\mathfrak{R}\left(\theta_{b(n)}\right) \geq 1$, when $b(n)=\{[0,0.1][0.1,0.3][0.3,0.5][0.5, \infty]\}$ from the center of the river representing transition from a high to low certainty. Table 3 shows $H R$ and FAR input values. An acceptable flood level when sandbag or levees are present can be set at $40 \mathrm{ft}$. The likelihood ratio of $l\left(\theta_{\mathrm{b}(0 \sim 0.1)}\right)$ is 122.3803 , which means that the distance penalty for travelling through the 0.1 mile buffer would be 122.3803-fold of the basic distance. In contrast, when the likelihood ratio of $l\left(\theta_{\mathrm{b}(0.5 \sim \infty)}\right)$ is 1.0, then no redundant resistance is charged for the roads in the over 0.5 mile buffer zone.

From Fig. 4, the $H R$ of the first category $\left(\theta_{b(0.0-0.1) \text { mile }}\right.$ buffer) shows a low hit rate of 0.31 in conjunction with a low FAR of 0.002 . These parameters increase for the second $\left(\theta_{b(0.1-0.3) \text { mile }}\right.$ buffer $)$ and third $\left(\theta_{b(0.3-0.5) \text { mile }}\right.$ buffer $)$ category concurrently. The sensitivity $P(A)$ overall shows a high proportion of $87 \%$ under the Receiver Operating Characteristic (ROC) curve (Fig. 4). This indicates that the index of the degree of overlap of the signal and noise distributions displays a high sensitivity with little overlapping. The bias of all the categories is $<1$, which means a risky criterion as set by a user when selecting a Yes (S) response. The likelihood of flood in each buffer was calculatedfrom:

$$
l\left(\theta_{b}\right)=\left\{\begin{array}{l}
\frac{P(S \mid s)}{P(S \mid n)} \text { if } l\left(\theta_{b}\right)>1 \\
1 \quad \text { otherwise }
\end{array}\right.
$$

From the normalization process, the road within the

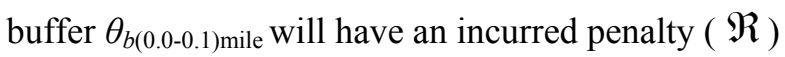

Table 3 SDT results based on assigned buffer zones.

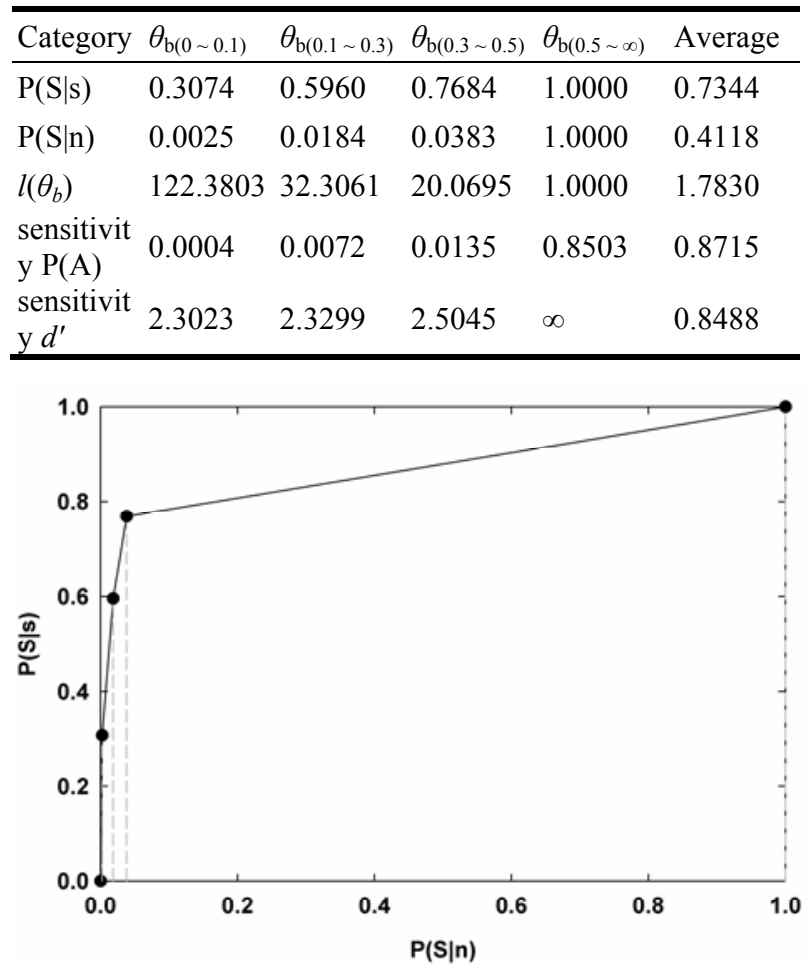

Fig. 4 Receiver Operating Characteristic (ROC) curve for assigned buffer categories. 
of about 122.38 times the length of a road segment, while those for the $\theta_{b(0.1-0.3) \text { mile }}$ and the $\theta_{b(0.3-0.5) \text { mile }}$ buffers will be $\times 32.31$ and $\times 20.06$ respectively.

Confidence rating can then be mapped into dichotomous yes-no categories and knowing that a set noise is continuous rather than binary then a finite space can be set. Therefore as $R\left(\theta_{h(n)}\right)=[0,1]$ and $\mathfrak{R}\left(\theta_{h(n)}\right) \geq 1$, then $h(n)=\{[0,30][30,35][35,40][40, \infty]\}$. Input values for elevation $h$ and response rare tabulated in Table 4. If the elevation is lower than 30 feet $(h=[0$, $30]$ ), we envision that this low area would surely be inundated due to the high likelihood contrary to $h=[40$, $\infty$ ]. From Table 4 , the hit rate of the first category $\left(\theta_{h(0-30) \text { feet }}\right)$ is higher at 0.52 with complementary low FAR value of 0.08 . A higher FAR of 0.97 and high HR of 0.91 was observed for $\theta_{h(30-35) \text { feet }}$ The likelihood ratio of over 40 feet water level is 1.00 which implies that any value falling below $\theta_{h(40-\infty) \text { feet }}$ would be considered safe, For example, $\theta_{h(30-40) \text { feet }}$ is below $\theta_{h(40-\infty) \text { feet }}$, so it would be safe to negotiate since FAR $>$ HR.If an observer drives through the region at $h_{(40-\infty) \text { feet }}$ takes a risk of magnitude $6.356-$ fold (that is, $0.5183 / 0.0816$ ).

In the same way, the sensitivity $P(A)$ of the third category shows a high proportion under the $R O C$ curve (Fig. 5) than the first and second categories. The total number of squares below the ROC curve represents $76 \%$ of all the regions. It implies that the index of the degree of overlap of the signal and noise distributions displays a high sensitivity with little overlapping. The likelihood of the first and second categorieswas applied to distance-weighted impedance (DWI) from Eq. (5). This was doneby multiplying the length of a road segments within each respective elevation class (rating categories). Values for the $\theta_{h(35-40) \text { feet }}$ and $\theta_{h(40-\infty) \text { feet }}$ categories were substituted by 1 to determine the resistance on segments in each elevation:

$$
l\left(\theta_{h}\right)= \begin{cases}\frac{P(S \mid s)}{P(S \mid n)} \text { if } l\left(\theta_{h}\right)>1 \\ 1 \quad \text { otherwise }\end{cases}
$$

Any impedance factors are void when $\omega=0.0$ (Fig. 6) by passing the closed bridge over the river and driving along the middle of the river. The route represents an ideal choice under normal operatingconditions. The routes are numerous and drivers can utilize any routes in the region to determine the fastestpath to a destination. The route choice disregards any water level and road capacity based on assigned classification. When $\omega=0.1$ then (1) the absolute resistance factors such as an impassable bridge, river area are

Table 4 SDT results based on assigned elevation zones.

\begin{tabular}{llllll}
\hline Category & $\theta_{\mathrm{h}(0 \sim 30)}$ & $\theta_{\mathrm{h}(30 \sim 35)}$ & $\theta_{\mathrm{h}(35 \sim 40)}$ & $\theta_{\mathrm{h}(40 \sim \infty)}$ & Average \\
\hline $\mathrm{P}(\mathrm{S} \mid \mathrm{s})$ & 0.5183 & 0.7273 & 0.9129 & 1.0000 & 0.8317 \\
$\mathrm{P}(\mathrm{S} \mid \mathrm{n})$ & 0.0816 & 0.1727 & 0.9656 & 1.0000 & 0.6440 \\
$l\left(\theta_{h}\right)$ & 6.3546 & 4.2123 & 0.9454 & 1.0000 & 1.2915 \\
sensitivit & 0.0211 & 0.0567 & 0.6503 & 0.0329 & 0.7610 \\
$\begin{array}{l}\text { y P(A) } \\
\text { sensitivit }\end{array}$ & 1.4405 & 1.5483 & -0.4611 & $\infty$ & 0.5918 \\
$\mathrm{y} d^{\prime}$ & & & & & \\
\hline
\end{tabular}

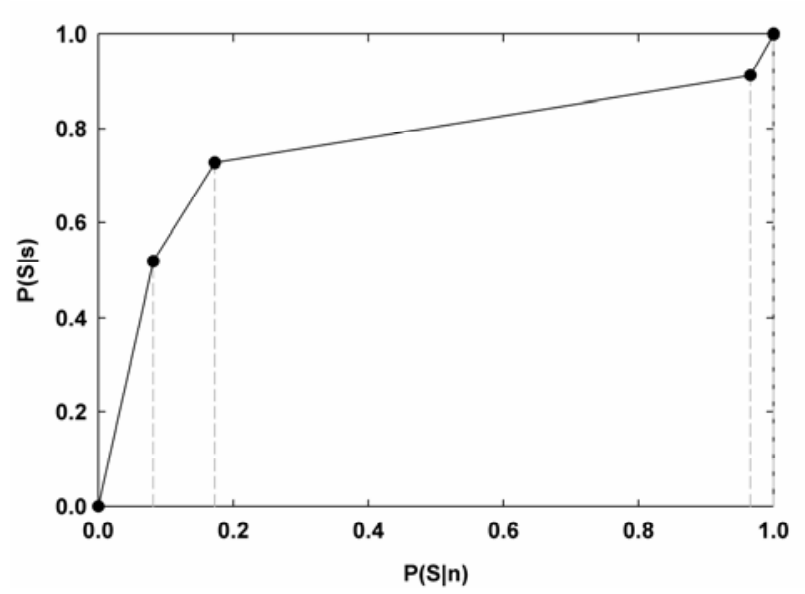

Fig. 5 Receiver Operating Characteristic (ROC) curve for assigned elevation categories.

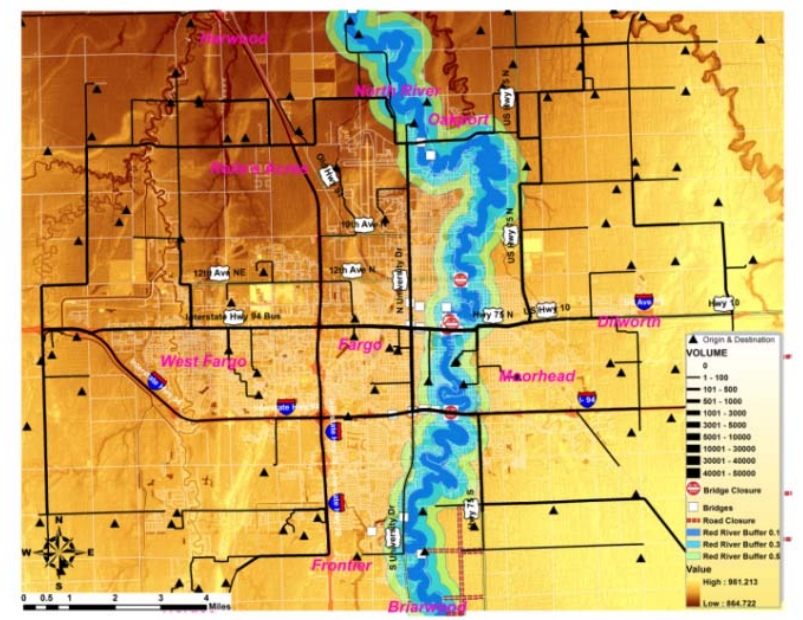

Fig. 6 Selected routes when $\omega=0.0$ corresponding to least impedance. 
systematically avoided, however, bridges in the downstream area can still be utilized, (2) the main routes are affected by bridge closures (see Fig. 7). Most travel is along the main artery right in the central area whereas north-south travel is along interstate highway I-29. $\omega=0.1$ represents a risk-takers' choice (fastest path considering the flood impedance factors).

Fig. 8 displays results for a risk-aversion choice, that is, $\omega=1.0$. In this case, even if the travel time is longer than a primary choice, a driver would readily select primary and/or secondary roads instead of urban and/or county roads. The algorithm developed creates a detour for a bridge crossing downstream. Comparing the risk-taker choice to the risk-averse choice (Figs. 7-8),

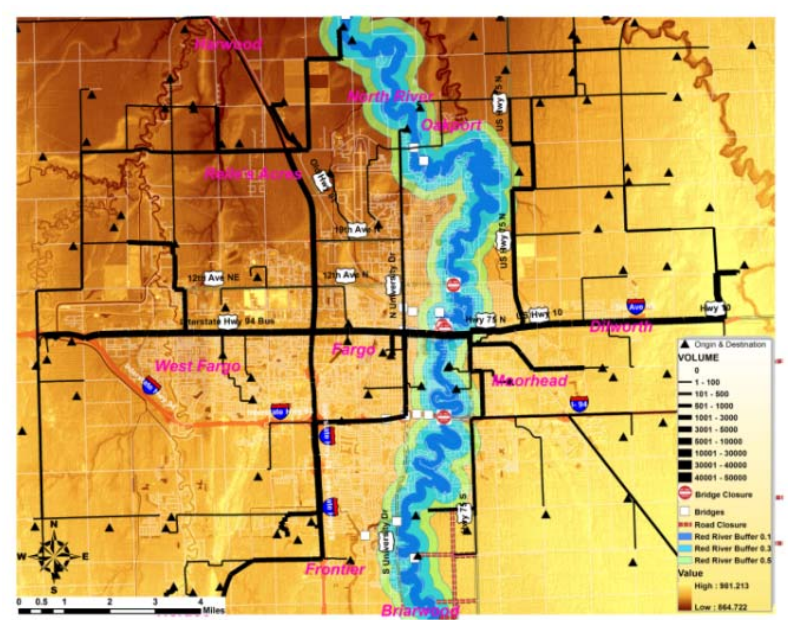

Fig. 7 Selected routes when $\omega=0.1$ corresponding to the risk-taker choice.

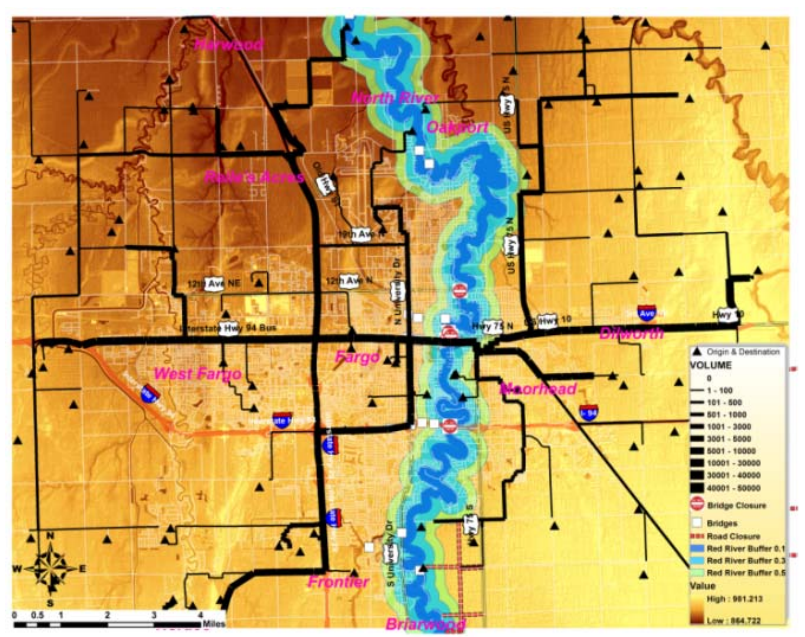

Fig. 8 Selected routes when $\omega=1.0$ corresponding to the risk-averse choice. the risk-takermay choose the riverside roads close to Oakport. Risk-averse travelers would rather take a road along the interstate highways.

In this study, the impedance is incremental (Fig. 9), that is, as the weight of ATT from Eq. 2 increases, the total impedance value increases for all routes. The 50 routes selected are grouped into three categories: significant change (33 routes), slight change (2 routes), and stable (15 routes). Especially, the route numbers 22 , 36,40 , and 42 indicates the grouping with the most significant change hence an increased sensitivity. On the other hand, routes 8 and 36 indicate only a comparative slight change.The differences between ten various weighting values for the routes were also analyzed. The equal means analysis resulted in differences within weights for $\mathrm{p}<0.005$. The change of each measurement of adjustment, time, and distance were within $90 \%$ and $95 \%$ confidence intervals. Pairwise comparisons were done to compare differences between two weights using ANOVA such that $\omega=0$ [27].

From Fig. 10, the impedance average does not significantly vary in response to weight. The trend depicted indicates continual variability increments, for example, for $\omega=0.6$ for routes 30 and $40 \Delta \mathrm{Ohm}=0.02$ similar to when $\omega=1.0$ for routes 6,8 , and 48 . Considering time (Fig. 11), the difference between $\omega=$ 0.0 and $\omega=0.1$ is significantly different. This is because at $\omega=0.0$ neither resistance nor reactance valuesis accounted. From Fig. 11, there is no significant mean differences when $\omega=0.2$ and $\omega=0.3$. There is however variation in confidence intervals for the interval $\omega=\{0.1,0.2,0.3,0.4\}$. In addition, it is not evident the mean time varies over $\omega=\{0.4,0.5,0.6$, $0.7,0.8,0.9,1.0\}$. Mean distance on the other hand varies significantly from $\omega=0.0$ to $\omega=0.1$ and from $\omega$ $=0.1$ to $\omega=0.2$ (Fig. 12) with little change at $\omega=0.2$ and $\omega=0.3$. The change from $\omega=0.5$ to $\omega=0.6$ indicates significant transition in all the routes. 


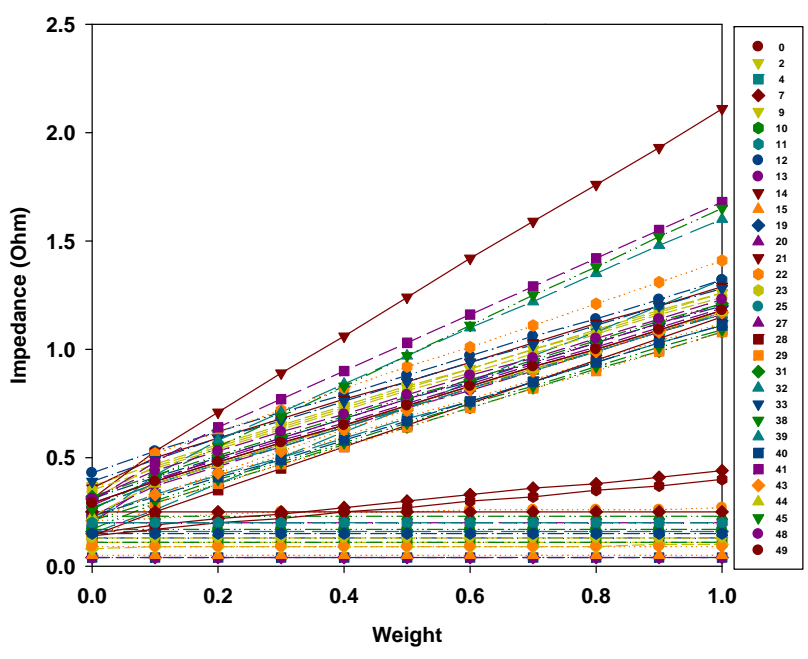

Fig. 9 Impedance $(\mathrm{Ohm})$ variation due to changing $\omega$.

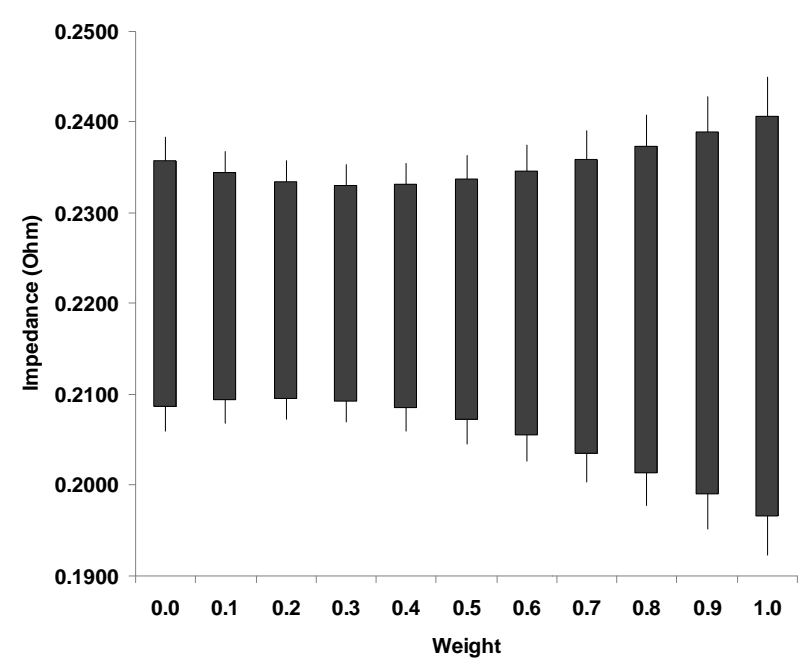

Fig. $10 \quad 90 \%$ and $95 \%$ confidence intervals for average Ohm.

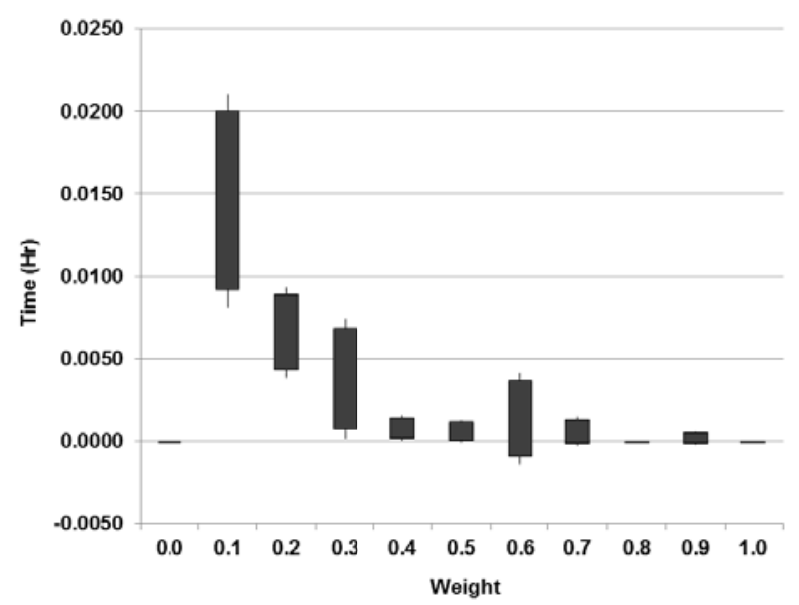

Fig. $1190 \%$ and $95 \%$ confidence intervals for average time (hours).

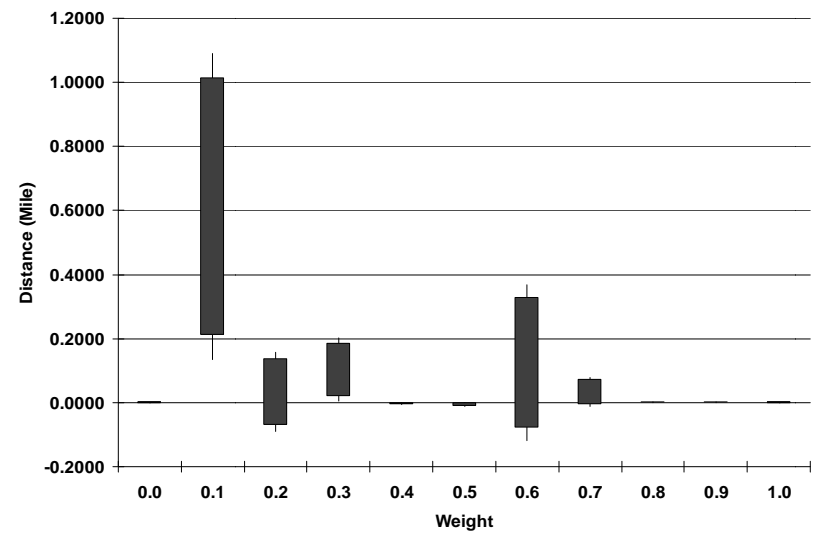

Fig. $1290 \%$ and $95 \%$ confidence intervals for average time distance (miles).

In summary (Fig. 13), both $\mathrm{Ohm}(\mathrm{Z})$ and time (Hr) show significant differences when $\omega=\{0.0,0.1,0.2\}$ whereas for distance this occurs only at $\omega=0.0$ and $\omega$ $=0.1$. There exists few route changes at $95 \%$ confidence interval for example, route number (ID = 46) displays a different time and distance due to higher weighting to the adjusted impedance denoting safe travel. At $\omega=0.0$ analysis indicates an unrealistic risk-taker since all closed bridges were assigned an absolute resistance. At $\omega=0.1$ and $\omega=0.2$ results displays the realistic risk-taker, one who is capable of taking side roads or detours, but avoids closed roads and bridges. From Fig. 13, by increasing $\omega$, most routes flatten out which can be interpreted as those that a risk-averse user would take. Overall results on $\omega$ values show polarization onto risk-taker and risk-averse users. An idealistic stage is achieved at $\omega=$ 0.4 or $\omega=0.5$, which indicates maximum achievement in terms of time gain and safety simultaneously. It is worthwhile to note that peak response times due to congestion from routes $0-50$ are assigned on a first-come-first-out (FIFO) allocation. Routes assigned later in this hierarchy would naturally be comparatively longer and associated segments would have higher impedances.

While the risk-averse avoid risky and unfavorable routes, the risk-taker optimizes at $\omega=0.1$ or $\omega=0.2$. At $\omega=0.0$ the prevailing conditions can be considered unrealistic since they incorporate areas considered 
impassable with absolute resistance like segments with a "Road Closed" or "Detour" sign. Nevertheless, this still provides a good source for sensitivity analysis and benchmarking for route choice behavioral inclination with pertinent resistance and reactance. The associatedselected routes that signify risk-prone routes are those that minimize traverse times with increasing related consequences. With $\omega=0.4$ or $\omega=0.5$, this yieldsan optimal grouping where the traverse time is compensated by choosing optimal distances and impedance. By increasing $\omega$, for example $\omega>0.5$, the system stabilizes with uniform impedance without significant changes of distance and travel time.

The user prediction programming module is normally based on available information for each travel route at the time of planning. In this study, this was incorporated using stochastic means, which provided

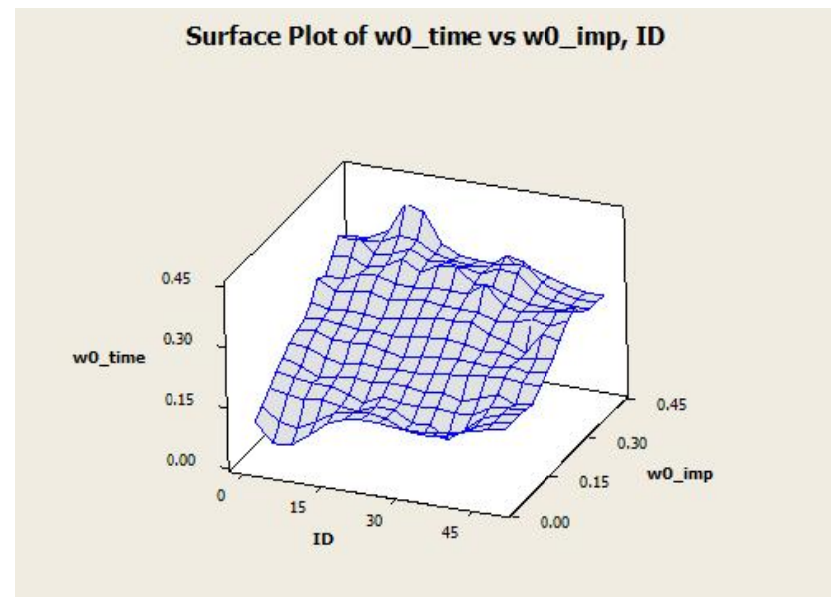

(a)

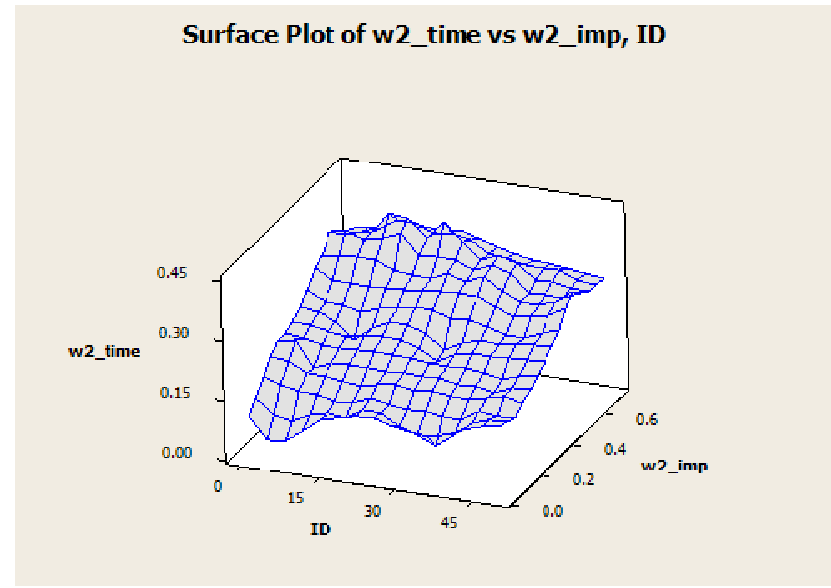

(c) ad hoc absolute resistance information (a) with or without user input and/or (b) from spatial parameters. When road links were located in an exact flood region (extremely strong signal), the roads were coded as definitely infeasible for travelling. However, the road segments in the buffer zones (response areas) may or may not be closed depending on a derived probability of inundation. If a warning signal is not confirmed over traverse time, signal from available surrounding sources were then used as benchmark information. To reflect a continuous stimulus under uncertainty, fuzzy signals and responses can be applied to represent the fuzziness of surrounding areas [4, 8, 28].

A 2009 flood area polygonal geospatial layer, Digital Elevation Models (DEM), and a mathematical inundation prediction model were used as basic information needed to estimate the areas that would be

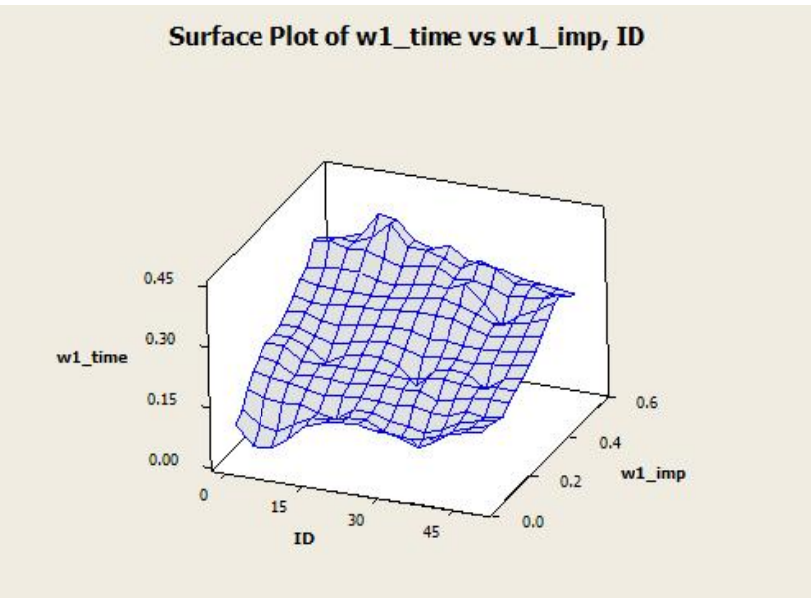

(b)

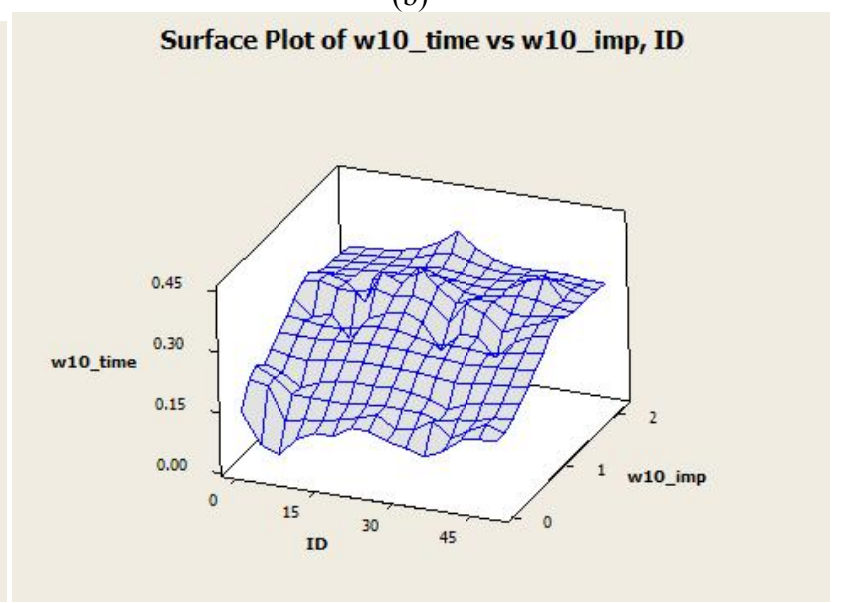

(d)

Fig. 13 Surface response graphs (a) $\omega=0.0$ (b) $\omega=0.1$, (c) $\omega=0.2$ and (d) $\omega=1.0$. 
significantly flooded including assigned areas where potential collateral damage may be experienced. Road and bridge closure information were also hard-coded within the algorithm. The proportions of hits and false alarms provide basic information; however, these may represent challenges in interpretation due to dichotomies of "Yes" or "No" responses.We were aware also that decision making is usually easier with a larger proportion of hits compared to false alarms [21].

Wachs and Kumangai [29] perceived that shortest-route travel time should be used when the actual impedance-driven travel time was not available. Measuring travel time in an urban area can be quite [30], so for a study like this, travel time in absence of resistance or reactance can be derived from the base travel speed based on TIGER $^{\circledR}$ network road classifications. The common measures of reactance disutility are traverse time, cost, and distance within network systems. The level of detail necessary to recognize and analyze reactance is based on data availability and system capability in data handling for any decision making [31]. When it comes to commuter mobility, the objective is to decrease travel times determined by a set number of delay hours [24]. Handy [32] used free-flow travel speeds to measure travel times during off-peak times. The free-flow speed ideally provides a state of minimum impedance or controls. Comparing to this study, minimum impedance still cannot yield zero impedance since even for a pristine system there is inherent impedance.

The most important process for costs-incurred estimation isspatial consistencyand contiguity for each impedance element (Road Classification, River Buffer, Elevation, and Congestion). First, the road or transportation network layer should be limited only within demarcated hazard zones in order to restrict route selection. Secondly, river buffers should only be generated based on flooded river regions. If flood polygon is generated from satellite imagery, then the pixels should be an appropriate spatial resolutions, extreme care is needed when subsampling or fusing raster data. We can also reiterate about the need for all spatial data to be at same datum, extent, coordinate system, and projection. Third, the elevation data needs to be at a fine resolution similar to any derived dataset or better. Fourth, distance units should be in SI units preferably.

In this study, signals were assumed fixed with respect to flood level and subsequent flood diminution was not accounted for from a reference data set. There are a couple of reasons for this kind of approach: (1) flooding signal can be consideredfuzzy and dynamic [4]. In other words, a flood signal varies over time and conditions can never be the same as predicted. To overcome this, we utilized publicly available and reasonable real-time information in our simulation. (2)Entities and people may react differently to a flooding event based on perception, for example, there exists contextual differences for low-area/high-risk and high-area/low-riskpairs.

\section{Conclusions}

We investigated the correlation between arisk-taking, risk-averse, or risk-neutralchoice and selected routes in a flooding scenario within Fargo-Moorhead area. Remotely sensed data and flood prediction modelswere utilized as inputs. From this study, we found out that Signal Detection Theory (SDT) improves the choice/route selectionanalysis by integrating basic signal benchmarking information with Geographic Information Systems (GIS). The main reasons for using SDT were two-fold: (1) to identify programmatically flooded routes based on applied signals; and (2) to ascertain route selection process and performance applying absolute and relative impedances.Remarkable concepts introduced by this study includes methods to aid in segregating between risk-takers' and risk-averse route selections using a multi-criteria route impedance approach for 50 randomly selected routes. The study shows sensitivity values for (1) $\omega=0.0$ to 0.3 grouping (2) $\omega=0.4$ to 0.5 , and (3) $\omega=0.6$ to 1.0. Conservative risk - averse with a 
weighting $\omega=1.0$, who practice safety conscience instead of quick response time, had longer travel times and slower speeds than average as expected.Route selection was executedusing the classical shortest path algorithm to minimize distance, time, and general physical link impedance.

\section{Acknowledgements}

The authors would like to acknowledge April Walaker from the City of Fargo and the City of Fargo Engineering DivisionGIS team for help in inundation prediction. We also would like to thank Grit May from the International Water Institute (IWI) for providing LiDAR data sets and help in deriving smoothed contours using various Python scripts.

\section{References}

[1] S. P. Simonovic and R. W. Carson, Flooding in the Red River Basin - Lessons from post flood activities, Natural Hazards 28 (2003) 345-365.

[2] S. P. Simonovic and S. Ahmad, Computer-based model for flood evacuation emergency planning, Natural Hazards 34 (2005) 25-51.

[3] D. Mioc, F. Antonand G. Liang, On-line street network analysis for flood evacuation planning, in: Nayak Shailesh and Zlatanova Sisi (Eds.), Remote Sensing and GIS Technologies for Monitoring and Prediction of Disasters, Springer, 2008.

[4] R. Parasuraman, J. A. Masalonis and A. P. Hancock, Fuzzy signal detection theory: Basic postulates and formulas for analyzing human and machine performance, Human Factors: The Journal of the Human Factors and Ergonomics Society 42 (4) (2000) 636-659.

[5] I. D. Cluckie, A. Moghaddamnia and D. Han, Practical hydroinformatics computational intelligence and technological developments in water applications, Springer, Berlin Heidelberg, 2008.

[6] T. A. Arentze and H. J. P. Timmermans, A learning-based transportation oriented simulation system, Transportation Research Part B: Methodological 38 (7) (2004) 613-633.

[7] R. P. Parasuraman, P. A. Hancock and O. Olofinboba, Alarm effectiveness in driver-centered collision-warning systems, Ergonomics 40 (3) (1997) 390-399.

[8] T. S. A. Wallis and M. S. Horswill, Using fuzzy signal detection theory to determine why experience and trained drivers respond faster than novices in a hazard perception test, Accident Analysis \& Prevention 39 (2007) 1177-1185.

[9] P. A. Hancock, A. J. Masalonis and R. Parasuraman, On the theory of fuzzy signal detection: Theoretical and practical considerations, Theoretical Issues in Ergonomic Science 1 (3) (2000) 207-230.

[10] B. Ivanov, Can your state recover from major disruptions to freight systems? Eight steps to resiliency, Federal Highway Administration Taking Freight Seminar, available online at: http://www.fhwa.dot.gov/ freightplanning/talking.htm, accessed on Dec. 20, 2009.

[11] J. Sohn, Evaluating the significance of highway network links under the flood damage: An accessibility approach, Transportation Research Part A 40 (2006) 491-506.

[12] A. Athanasenas, Traffic simulation models for rural road network management, Transportation Research Part E 33 (3) (1997) 233-243.

[13] E. Lee and K. Farahmand, Considering impedance for intermodal freight transportation, in: Proceedings of 2009 Institute of Industrial Engineering Annual Conference, Miami, FL. May 30 - June 3, 2009, pp. 1345-1350.

[14] Oak Ridge National Laboratory, Railroad network, Center for Transportation Analysis, March 2004, available online at: http://cta.ornl.gov/transnet /RailRoads.html, accessed March 13, 2009.

[15] F. Southworth and B. E. Peterson, Intermodal and international freight network modeling, Transportation Research Part C 8 (2000) 147-166.

[16] S. S. Keshkamat, J. M. Looijen and M. H. P. Zuidgeest, The formulation and evaluation of transport route planning alternatives: A spatial decision support system for the via Baltica project, Poland, Journal of Transport Geography 17 (1) (2009) 54-64.

[17] Transportation Research Board, Highway Capacity Manual (HCM) 2000, National Research Council, Washington, D.C., 2000.

[18] M. Alam, E. Fekpe and M. Majed, FAF freight traffic analysis: Freight management and operations, Federal Highway Administration, U.S. Department of Transportation, Washington D.C., 2007.

[19] J. P. Rodrigue, C. Comtois and B. Slack, The geography of transport systems, Routledge, New York, 2006.

[20] J. A. Swets, W. P. Tanner and T. G. Birdsall, Decision process in perception, Psychological Review 68 (5) (1961) 301-340.

[21] D. McNicol, A primer of signal detection theory, Lawrence Erlbaum Associates, Publisher, Mahwah, New Jersey, 2005.

[22] B. McGuiness, Quantitative analysis of situational awareness (QUASA): Applying signal detection theory to true/false probes and self-ratings, in: Proceedings of 2004 


\section{Application of Signal Detection Theory to Ascertain Driving Risk and Mitigation in a Flooded Urban Area}

Command and Control Research and Technology Symposium, Copenhagen, Sep. 2004.

[23] M. S. Sanders, Human Factors in Engineering and Design, McGraw-Hill, Inc., 1993, pp. 62-65.

[24] Transportation Research Board, Guide to Effective Freeway Performance Measurement: Final Report and Guide Book, National Cooperative Highway Research Program, Transportation Research Board of the National Academies, Washington D.C., 2006.

[25] H. J. Miller, Measuring space-time accessibility benefits within transportation networks: basic theory and computational procedures, Geographical Analysis 31 (1999) $187-212$.

[26] T. Grossardt, K. Bailey and J. Barumm, Analytic minimum impedance surface: Geographic Information System-based corridor planning methodology, Transportation Research Record No. 1768, Paper No. (01-2579), 2001, pp. 224-232.
[27] W. Mendenhall and T. Sincich, A Second Course Statistics: Regression Analysis (6th ed.), Peason Education Inc., Upper Saddle River, NJ, USA, 2003.

[28] T. Chen and J. M. J. Wang, Applying fuzzy set approach to signal detection theory, Fuzzy Sets and Systems 72 (1995) 39-49.

[29] M. Wachs and T. G. Kumangai, Physical accessibility as a social indicator, Socio-Economic Planning Science 7 (5) (1973) 437-456.

[30] C. Woudsma, Understanding the movement of goods, not people: Issues, evidence and potential, Urban Studies 38 (13) (2001) 2439-2455.

[31] K. T. Geurs and B. V. Wee, Accessibility evaluation of land-use and transport strategies: Review and research directions, Journal of Transport Geography 12 (2004) 127-140.

[32] S. L. Handy, Regional versus local accessibility: Implications for nonwork travel, Transportation Research Record 1400 (1993) 58-66. 